\title{
Markers of metabolic health and gut microbiome diversity: findings from two population-based cohort studies
}

\author{
Semi Zouiouich ${ }^{1}$ (D) Erikka Loftfield ${ }^{2} \cdot$ Inge Huybrechts $^{1} \cdot$ Vivian Viallon $^{1} \cdot$ Panayiotis Louca $^{3} \cdot$ Emily Vogtmann $^{2}$. \\ Philippa M. Wells ${ }^{3}$ - Claire J. Steves ${ }^{3}$ - Karl-Heinz Herzig ${ }^{4,5}$ - Cristina Menni ${ }^{3}$ - Marjo-Riitta Jarvelin ${ }^{6,7,8,9}$. \\ Rashmi Sinha ${ }^{2} \cdot$ Marc J. Gunter $^{1}$
}

Received: 28 September 2020 / Accepted: 8 March 2021 / Published online: 10 June 2021

(C) The Author(s) 2021

\begin{abstract}
Aims/hypothesis The gut microbiome is hypothesised to be related to insulin resistance and other metabolic variables. However, data from population-based studies are limited. We investigated associations between serologic measures of metabolic health and the gut microbiome in the Northern Finland Birth Cohort 1966 (NFBC1966) and the TwinsUK cohort.

Methods Among 506 individuals from the NFBC1966 with available faecal microbiome (16S rRNA gene sequence) data, we estimated associations between gut microbiome diversity metrics and serologic levels of HOMA for insulin resistance (HOMAIR), $\mathrm{HbA}_{1 \mathrm{c}}$ and $\mathrm{C}$-reactive protein (CRP) using multivariable linear regression models adjusted for sex, smoking status and BMI. Associations between gut microbiome diversity measures and HOMA-IR and CRP were replicated in 1140 adult participants from TwinsUK, with available faecal microbiome (16S rRNA gene sequence) data. For both cohorts, we used general linear models with a quasi-Poisson distribution and Microbiome Regression-based Kernel Association Test (MiRKAT) to estimate associations of metabolic variables with alpha- and beta diversity metrics, respectively, and generalised additive models for location scale and shape (GAMLSS) fitted with the zero-inflated beta distribution to identify taxa associated with the metabolic markers.

Results In NFBC1966, alpha diversity was lower in individuals with higher HOMA-IR with a mean of 74.4 (95\% CI 70.7, 78.3) amplicon sequence variants (ASVs) for the first quartile of HOMA-IR and 66.6 (95\% CI 62.9, 70.4) for the fourth quartile of HOMA-IR. Alpha diversity was also lower with higher $\mathrm{HbA}_{1 \mathrm{c}}$ (number of ASVs and Shannon's diversity, $p<0.001$ and $p=$ 0.003 , respectively) and higher CRP (number of ASVs, $p=0.025$ ), even after adjustment for BMI and other potential confounders. In TwinsUK, alpha diversity measures were also lower among participants with higher measures of HOMA-IR and CRP. When considering beta diversity measures, we found that microbial community profiles were associated with HOMAIR in NFBC1966 and TwinsUK, using multivariate MiRKAT models, with binomial deviance dissimilarity $p$ values of $<0.001$. In GAMLSS models, the relative abundances of individual genera Prevotella and Blautia were associated with HOMA-IR in both cohorts.
\end{abstract}

Semi Zouiouich

semi.zouiouich@nih.gov

1 Section of Nutrition and Metabolism, International Agency for Research on Cancer-WHO, Lyon, France

2 Metabolic Epidemiology Branch, Division of Cancer Epidemiology and Genetics, National Cancer Institute, Rockville, MD, USA

3 Department of Twin Research, King's College London, London, UK

4 Research Unit of Biomedicine, Medical Research Center (MRC), University of Oulu, University Hospital, Oulu, Finland
5 Department of Gastroenterology and Metabolism, Poznan University of Medical Sciences, Poznan, Poland

6 Department of Epidemiology and Biostatistics, MRC-PHE Centre for Environment and Health, School of Public Health, Imperial College London, London, UK

7 Center for Life Course Health Research, Faculty of Medicine, University of Oulu, Oulu, Finland

8 Unit of Primary Health Care, Oulu University Hospital, OYS, Oulu, Finland

9 Department of Life Sciences, College of Health and Life Sciences, Brunel University London, London, UK 


\section{Research in context}

\section{What is already known about this subject?}

- Insulin resistance lies on the causal pathways of diabetes and other metabolic diseases; however, the pathophysiological origins of insulin resistance are not fully understood

- The gut microbiome has been hypothesised to influence the development of insulin resistance and the host metabolic state may also impact the gut microbiota. However, human data on insulin resistance and the gut microbiome are limited as very few population-based cohorts have collected both blood and faecal specimens

\section{What is the key question?}

- Is there an association between the gut microbiome and serologic measures of metabolic health in population-based studies?

\section{What are the new findings?}

- Higher levels of insulin resistance and other markers of metabolic dysfunction were associated with lower diversity of microbiome composition in two population-based cohort studies, even after adjustment for obesity

\section{How might this impact on clinical practice in the foreseeable future?}

- This study suggests that individuals with worse metabolic control may have lower gut microbial diversity. The potential causality of these results needs to be further explored in mechanistic and longitudinal studies

Conclusions/interpretation Overall, higher levels of HOMA-IR, CRP and $\mathrm{HbA}_{1 \mathrm{c}}$ were associated with lower microbiome diversity in both the NFBC1966 and TwinsUK cohorts, even after adjustment for BMI and other variables. These results from two distinct population-based cohorts provide evidence for an association between metabolic variables and gut microbial diversity. Further experimental and mechanistic insights are now needed to provide understanding of the potential causal mechanisms that may link the gut microbiota with metabolic health.

Keywords Faecal microbiome $\cdot$ HOMA-IR $\cdot$ Insulin resistance $\cdot$ Metabolic health

$\begin{array}{ll}\text { Abbreviations } \\ \text { ASV } & \text { Amplicon sequence variants } \\ \text { BCAA } & \text { Branched-chain amino acids } \\ \text { CRP } & \text { C-reactive protein } \\ \text { FDR } & \text { False discovery rate } \\ \text { GAMLSS } & \begin{array}{l}\text { Generalised additive models for } \\ \text { location scale and shape }\end{array} \\ \text { MiRKAT } & \begin{array}{l}\text { Microbiome Regression-based } \\ \text { Kernel Association Test }\end{array} \\ \text { NFBC1966 } & \begin{array}{l}\text { Northern Finland Birth Cohort } \\ \text { VIF }\end{array} \\ & \text { Variance inflation factors }\end{array}$

\section{Introduction}

Obesity and type 2 diabetes have reached global epidemic proportions and are recognised as major causes of morbidity and mortality [1,2]. Insulin resistance is a pathophysiological condition that precedes the development of type 2 diabetes [3]; however, its aetiology is not fully understood. Obesity is a recognised risk factor for insulin resistance but not all insulin-resistant individuals are overweight or obese [4]; indeed, the existence of metabolically healthy obese and metabolically unhealthy normal weight individuals has been described in previous studies [5]. Despite the wellestablished epidemiological links between insulin resistance, its related variables (i.e., poor glucose control and high levels of inflammation) and chronic diseases including obesity and type 2 diabetes, the potential role of the gut microbiome in the development of insulin resistance and type 2 diabetes is not fully understood.

Previous studies have suggested a link between the gut microbiome and metabolic health [6,7], and have described differences of microbial composition and functionality in type 2 diabetes patients compared with healthy participants $[7,8]$. The gut microbiome is potentially 
associated with host metabolic health through several pathways including energy extraction, intestinal barrier integrity, metabolism of bile acids and host metabolic and signalling pathways, which are directly or indirectly related to insulin resistance development [9]. For example, circulating levels of branched-chain amino acids (BCAA) have been positively associated with insulin resistance [10] and BCAA levels in insulin-resistant individuals correlate with specific changes in gut microbiome composition and functions, such as enriched potential for BCAA biosynthesis and deprivation of genes encoding for BCAA transport into bacterial cells [11]. However, because of the cross-sectional nature of these studies, it is impossible to conclude whether these differences in microbial composition are a cause or a consequence of metabolic dysfunction.

Despite an important expansion of research on the relationship between the human gut microbiome and metabolic disorders in recent years, human data on the link between the gut microbiota and insulin resistance from population-based studies are lacking. In this study, we investigated the relationship between measures of metabolic health (i.e., insulin resistance, glucose control and inflammation) and gut microbial diversity, independent of BMI, among individuals in the Northern Finland Birth Cohort (NFBC1966) and then sought to replicate these findings in the TwinsUK cohort.

\section{Methods}

\section{Ethics approval and consent to participate}

Informed written consent was obtained from all NFBC1966 participants, and the research protocols were approved by the Ethics Committee of Northern Ostrobotnia Hospital District, Finland. Written informed consent was obtained from all the TwinUK volunteers upon registration and also during their clinical visits, and the research protocols had ethical approval as part of the TwinsUK (EC04/015) study from the Local Research Ethics Committee at the Department of Twin Research and Genetic Epidemiology, King's College London. The study was approved by the International Agency for Research on Cancer Ethics Committee.

\section{Northern Finland Birth Cohort}

Study design and samples The NFBC1966 was established in 1965 and included 12,055 pregnant women with expected delivery dates between 1 January and 31 December 1966, and subsequently 12,058 children representing $96 \%$ of live births in two Finnish provinces, Oulu and Lapland (https:// www.oulu.fi/NFBC1966/) [12]. Pregnancies were followed prospectively and children were followed through childhood, adolescence and early adulthood up to 46 years of age. At 31 and 46 years of age, information about health, behaviour, work and social background were collected using self-administered questionnaires and clinical examinations were performed. At 31 and 46 years of age, measures of metabolic health were generated using blood samples. Additionally, at 46 years of age, the participants were asked to collect a stool sample at home using a collection tube without additive that was provided by study investigators. Participants were asked to return the sample to the study facility the same day; if the specimen was taken 1-2 days in advance, the participants were asked to store the stool sample at $-20^{\circ} \mathrm{C}$. At the study laboratory, the stool samples were first transferred to $-20^{\circ} \mathrm{C}$ freezers and after, to long-term $-70^{\circ} \mathrm{C}$ freezers without any additive within days of collection.

This investigation builds upon a related study conducted by Loftfield et al within the NFBC1966, which aimed to explore associations of BMI history and adult BMI with faecal microbial diversity $(n=565)$ and microbial metabolite levels $(n=$ 340) [13]. In the current analysis, participants were selected from the same cohort with faecal microbial diversity data and were included if measures of metabolic health (i.e., insulin $[\mu \mathrm{U} /$ $\mathrm{ml}]$, glucose [mmol/l], high-sensitivity C-reactive protein $[\mathrm{mg} /$ 1], and $\left.\mathrm{HbA}_{1 \mathrm{c}}[\mathrm{mmol} / \mathrm{mol}]\right)$ were available at 46 years of age. In total, 506 participants were included in our analytic sample, comprising 187 men (37.0\%) and 319 women (63.0\%).

At age 46 years, participants underwent clinical examination, during which height and weight were measured, and they completed self-administered questionnaires reporting lifestyle and demographic characteristics. BMI was estimated as measured weight $(\mathrm{kg})$ divided by measured height $(\mathrm{m})$ squared $\left(\mathrm{kg} / \mathrm{m}^{2}\right)$. BMI was categorised according to the WHO international classification system: normal weight (18.5-24.9), overweight/pre-obese (25.0-29.9), obese $(\geq$ $30.0)$. Smoking status was categorised as never, former or current smoker.

Laboratory methods The laboratory methods have previously been described in detail [14]. Metabolic markers were measured for all individuals after an overnight fasting period (12 h). Fasting plasma glucose was analysed by an enzymatic dehydrogenase method (Advia 1800, Siemens Healthcare Diagnostics, Tarrytown, New York, USA). Fasting serum insulin was analysed by a chemiluminometric immunoassay (Advia Centaur XP, Siemens Healthcare Diagnostics, Tarrytown, New York, USA). HOMA for insulin resistance (HOMA-IR) is a commonly used measure of insulin resistance and is calculated as previously described [15]. HOMA-IR values were subdivided into quartiles based on their distribution across all included participants: $\mathrm{Q}_{1}:<1.20$, $\mathrm{Q}_{2}: 1.20-1.91, \mathrm{Q}_{3}: 1.92-3.1, \mathrm{Q}_{4}:>3.11$ for categorical analyses. The concentrations of $\mathrm{HbA}_{1 \mathrm{c}}$ were measured using 
immunochemical assay methods. High-sensitivity C-reactive protein (CRP) was analysed by an immune-nephelometric assay (BN ProSpec, Siemens Healthcare Diagnostics, Newark, Delaware, USA). HOMA-IR, $\mathrm{HbA}_{1 \mathrm{c}}$ and CRP were log-transformed (natural logarithm) for continuous analyses.

DNA extraction, amplification and sequencing Faecal samples were processed at the University of California, San Diego (La Jolla, California). DNA extraction, PCR amplification and sequencing were completed as described by Loftfield et al [13] and by Vogtmann et al [16], using the universal bacterial primer set 515F/806R [17]. In brief, study samples were randomly ordered and distributed within the batches. For technical reproducibility, replicate faecal samples from three individuals $(n=62)$ were distributed within and across batches. Four quality control samples were also included within each DNA extraction batch: artificial community, chemostat community [18], extraction blank and PCR blank. DNA was extracted with the MO-BIO PowerSoil DNA isolation kit and the V4 region of the 16S rRNA gene was PCR amplified and $2 \times 150$ bp paired-end sequencing was performed on the MiSeq (Illumina, San Diego, CA). After removing singletons and reads with read errors, the mean coverage was approximately 112,000 reads per sample.

Bioinformatics Processing was performed using QIIME 2 2017.8 [19]. Sequences were demultiplexed, and quality control on forward reads was performed with DADA2 [20]. Paired-end reads were not joined because shorter 16S rRNA gene sequences would be dropped, resulting in systematic bias in community composition. Taxonomy was assigned to amplicon sequence variants (ASVs) using q2-feature-classifier [21] and the Greengenes 13_8 reference database [22]. A phylogenetic tree was built by aligning ASVs with MAFFT [23], filtering highly variable positions via q2-alignment, and applying FastTree [24] to construct an unrooted tree, followed by midpoint rooting using q2-phylogeny midpoint-root. Diversity metrics (i.e., Shannon index, observed sequence variants, binomial deviance dissimilarity, Jaccard, weighted and unweighted UniFrac) were computed using the 'vegan' [25] and 'microbiome' packages in R (version 3.6.0) at a depth of 10,000 reads per sample to represent the diversity of unique sequences in each sample. As described by Lofffield et al, inspection of quality control data suggested good reproducibility within and across batches [13].

\section{TwinsUK}

Study design and samples The design of the TwinsUK cohort has been described previously [26]. In brief, the TwinsUK cohort is one of the largest adult twin registries comprising over 14,000 volunteers followed over more than two decades. Participants were predominantly female $(>80 \%)$ and middleaged (mean age 59). Data were collected during visits to the
Department of Twin Research and Genetic Epidemiology, King's College London, resulting in biochemical, behavioural, dietary and socioeconomic characterisation. In the current study, we analysed data on the gut microbiome and both HOMA-IR and CRP from 1140 female participants. Anthropometric measurements, including height and weight, were measured during each participant's annual clinic visit, allowing BMI to be calculated. Questionnaires were used to define age (from birthdate) and smoking status.

Laboratory methods Plasma glucose and insulin were measured for all individuals after a $10 \mathrm{~h}$ overnight fast. Insulin was quantified by immunoassay (Abbott Laboratories, Maidenhead, UK) and glucose was measured by an Ektachem 700 multichannel analyser using an enzymatic colorimetric slide assay (Johnson and Johnson Clinical Diagnostic Systems, Amersham, UK), as previously described [27]. Highly sensitive CRP was measured by latex-enhanced nephelometry on a Siemens Prospec Nephelometer. HOMA-IR was calculated as described above for NFBC1966. HOMA-IR, $\mathrm{HbA}_{1 \mathrm{c}}$ and CRP were logtransformed (natural logarithm) for continuous analyses.

DNA extraction, sequencing and bioinformatics Faecal samples were collected at home and brought or sent on ice to the clinical research facility where they were stored at $-80^{\circ} \mathrm{C}$. Samples were then processed to determine gut microbial composition by 16S rRNA gene sequencing, as previously described [28]. Briefly, amplicon PCR was performed on the $\mathrm{V} 4$ region of the $16 \mathrm{~S}$ rRNA gene using the primer pair $515 \mathrm{f}$ to 806r with Golay error-correcting barcodes on the reverse primer. The barcoded amplicon pool was purified with the MO-BIO UltraClean PCR cleanup kit and sequenced on the Illumina MiSeq platform. Sequence data were demultiplexed using the QIIME2 2017.8 [19]. ASVs were generated with DADA2 [20]. Diversity metrics (i.e., Shannon index, observed sequence variants, binomial deviance dissimilarity, Jaccard, weighted and unweighted UniFrac) are presented in electronic supplementary material (ESM) Table 1 and were computed using the 'vegan' (25) and 'microbiome' packages in $\mathrm{R}$ (verion 3.6.0).

\section{Statistical analyses}

Descriptive characteristics of the participants were presented by BMI groups. As presented in Table 1, the majority of NFBC1966 participants were female, and $29.2 \%, 45.9 \%$ and $24.9 \%$ were normal weight, overweight and obese, respectively. The TwinsUK cohort was entirely female and older than the NFBC1966 cohort (mean age 62.9 and 46.6 years, respectively). Although the NFBC1966 results presented here include both men and women, sensitivity analyses were performed in women only $(n=319)$ with similar results obtained (data 
Table 1 Description of the study population stratified by BMI category in NFBC1966 and TwinsUK

\begin{tabular}{|c|c|c|c|c|c|c|}
\hline \multirow[b]{3}{*}{ Variable } & \multicolumn{3}{|c|}{ NFBC1966 $(n=506)$} & \multicolumn{3}{|c|}{ TwinsUK ( $n=1140)$} \\
\hline & \multicolumn{3}{|l|}{ BMI, $\mathrm{kg} / \mathrm{m}^{2}$} & \multicolumn{3}{|l|}{ BMI, $\mathrm{kg} / \mathrm{m}^{2}$} \\
\hline & 18.5 to 24.9 & 25.0 to 29.9 & over 30 & 18.5 to 24.9 & 25.0 to 29.9 & over 30 \\
\hline $\begin{array}{l}\text { Total study } \\
\text { population }\end{array}$ & $148(29.2)$ & $232(45.9)$ & $126(24.9)$ & $499(43.8)$ & $411(36.1)$ & $230(20.2)$ \\
\hline Male & $32(17.1)$ & $108(57.8)$ & $47(25.1)$ & 0 & 0 & 0 \\
\hline Female & $116(36.4)$ & $124(38.9)$ & $79(24.8)$ & 499 (43.7) & $411(36.1)$ & $230(20.2)$ \\
\hline Age, years & $46.6 \pm 0.6$ & $46.6 \pm 0.5$ & $46.7 \pm 0.6$ & $62.9 \pm 8.9$ & $64.2 \pm 8.5$ & $61.8 \pm 8.4$ \\
\hline Never smoker & $98(33.9)$ & $128(44.3)$ & $63(21.8)$ & $300(43.7)$ & $235(34.2)$ & $152(22.1)$ \\
\hline Former smoker & $29(23.6)$ & $61(49.6)$ & $33(26.8)$ & $152(45.0)$ & 127 (37.6) & $59(17.5)$ \\
\hline Current smoker & $21(22.3)$ & $43(45.8)$ & $30(31.9)$ & $47(40.9)$ & $49(42.6)$ & $19(16.5)$ \\
\hline HOMA-IR & $1.32 \pm 0.7$ & $2.26 \pm 1.3$ & $4.61 \pm 4.4$ & $0.84 \pm 1.32$ & $1.12 \pm 1.46$ & $1.56 \pm 1.37$ \\
\hline CRP, mg/l & $1.08 \pm 1.7$ & $1.45 \pm 2.7$ & $2.95 \pm 6.6$ & $1.88 \pm 2.95$ & $2.89 \pm 6.29$ & $5.17 \pm 8.41$ \\
\hline $\mathrm{HbA}_{1 \mathrm{c}}, \mathrm{mmol} / \mathrm{mol}$ & $33.20 \pm 2.0$ & $34.02 \pm 4.2$ & $35.03 \pm 3.6$ & - & - & - \\
\hline $\mathrm{HbA}_{1 \mathrm{c}}, \%$ & 5.2 & 5.3 & 5.4 & - & - & - \\
\hline
\end{tabular}

Data are shown as $n(\%)$ or mean $\pm \mathrm{SD}$ not shown). In NFBC1966, 31.9\% of current smokers were obese vs $16.5 \%$ in TwinsUK.

Spearman correlations were calculated to estimate correlations between the different measures of metabolic health and BMI. In NFBC1966, BMI was strongly correlated with HOMA-IR $(R=0.64 ; 95 \%$ CI $0.58,0.68)$ and more moderately correlated with CRP $(R=0.37 ; 95 \%$ CI $0.29,0.44)$ and $\mathrm{HbA}_{1 \mathrm{c}}(R=0.23 ; 95 \%$ CI $0.14,0.31)$ (ESM Fig. 1$)$. In TwinsUK, BMI was moderately correlated with HOMA-IR $(R=0.43 ; 95 \%$ CI $0.38,0.47)$ and moderately correlated with CRP $(R=0.37 ; 95 \%$ CI $0.31,0.41)$. Prior to conducting multivariable analyses, variance inflation factors (VIF) were calculated to evaluate potential multicollinearity between alpha diversity metrics, BMI and HOMA; moderate VIF were found with these two covariates (VIF BMI 1.37, VIF HOMA-IR $1.43)$, suggesting that these predictors are not correlated with other variables. To estimate the association of the measures of metabolic health as categorical and continuous variables with the alpha diversity metrics, general linear models with a quasiPoisson distribution were used. The estimated value in Table 2 signifies how much the mean of the dependent variable (measures of microbiome diversity) changes given a oneunit shift in the independent variable (markers of metabolic health) while holding other variables in the model constant. In the NFBC1966, no visual clustering was observed by measures of metabolic health using the first three principal coordinate analysis vectors from four beta diversity matrices (results not shown). For the association between microbial community profiles using beta diversity metrics and the measures of metabolic health, MiRKAT tests were performed [29]. To identify the taxa associated with HOMA-IR, CRP and $\mathrm{HbA}_{1 \mathrm{c}}$, after grouping ASVs at the genus level, a generalised additive model for location scale and shape (GAMLSS) fitted with the zero-inflated beta distribution was computed using the 'gamlss' package in R [30]. Likelihoodratio tests between models including adjustment factors only and models including the measures of metabolic health and adjustment factors were performed. $P$ values $<0.05$ were considered as indicators of an association between metabolic health and distribution of the taxonomic component. The $p$ values were then adjusted using a Benjamini-Hochberg false discovery rate (FDR) $<0.05$. All statistical tests described above were adjusted for BMI, sex and smoking status in the NFBC1966 cohort and for BMI, age and smoking status in the TwinsUK cohort. Other possible confounders (i.e., education, alcohol, fruit, vegetables, cereals, fish, red and processed meat, poultry, dairy and physical activity) were considered but not selected in the final models, based on a bidirectional stepwise selection.

\section{Results}

\section{Alpha diversity}

Overall, alpha diversity declined with increasing quartiles of HOMA-IR in the NFBC1966 (Table 2). For example, the mean number of ASVs was lower in the 4th quartile of HOMA-IR $(66.6,95 \%$ CI $62.9,70.4)$ compared with the 1st quartile $(74.4,95 \%$ CI 70.7, 78.3) (ESM Table 2). Similar results were found in the TwinsUK cohort where Shannon's diversity was lower in the 4th quartile of HOMA-IR (3.72, $95 \%$ CI $3.65,3.79)$ than the 1 st quartile $(3.91,95 \%$ CI 3.84 , 3.98) (ESM Table 2). In NFBC1966, the number of observed ASVs was inversely associated with HOMA-IR (estimate per 
Table 2 Associations of metabolic variables (continuous and categorical) with measures of alpha diversity in NFBC1966 and TwinsUK

\begin{tabular}{|c|c|c|c|c|c|c|c|c|}
\hline \multirow[b]{3}{*}{ Variable } & \multicolumn{4}{|c|}{$\operatorname{NFBC} 1966(\mathrm{n}=506)^{\mathrm{a}}$} & \multicolumn{4}{|c|}{ TwinsUK $(\mathrm{n}=1140)^{\mathrm{b}}$} \\
\hline & \multicolumn{2}{|c|}{ Shannon's diversity } & \multicolumn{2}{|c|}{ Observed ASVs } & \multicolumn{2}{|c|}{ Shannon's diversity } & \multicolumn{2}{|c|}{ Observed ASVs } \\
\hline & Estimate & $p$ value & Estimate & $p$ value & Estimate & $p$ value & Estimate & $p$ value \\
\hline \multicolumn{9}{|l|}{ HOMA-IR } \\
\hline Continuous & -0.047 & 0.001 & -0.072 & 0.002 & -0.062 & $<0.001$ & -0.106 & 0.001 \\
\hline Quartile 1 & Ref & Ref & Ref & Ref & Ref & Ref & Ref & Ref \\
\hline Quartile 2 & -0.022 & 0.282 & -0.033 & 0.304 & -0.021 & 0.051 & -0.019 & 0.413 \\
\hline Quartile 3 & -0.019 & 0.379 & -0.055 & 0.118 & -0.027 & 0.017 & -0.041 & 0.083 \\
\hline Quartile 4 & -0.078 & 0.003 & -0.111 & 0.008 & -0.055 & $<0.001$ & -0.093 & $<0.001$ \\
\hline \multicolumn{9}{|l|}{ CRP } \\
\hline Continuous & -0.016 & 0.085 & -0.032 & 0.025 & -0.018 & 0.031 & -0.041 & 0.019 \\
\hline Quartile 1 & Ref & Ref & Ref & Ref & Ref & Ref & Ref & Ref \\
\hline Quartile 2 & -0.031 & 0.154 & -0.007 & 0.843 & -0.007 & 0.523 & -0.01 & 0.657 \\
\hline Quartile 3 & -0.023 & 0.303 & -0.028 & 0.415 & -0.006 & 0.599 & -0.009 & 0.688 \\
\hline Quartile 4 & -0.027 & 0.263 & -0.049 & 0.195 & -0.028 & 0.016 & -0.063 & 0.01 \\
\hline \multicolumn{9}{|l|}{$\mathrm{HbA}_{1 \mathrm{c}}$} \\
\hline Continuous & -0.042 & 0.003 & -0.113 & $<0.001$ & - & - & - & - \\
\hline Quartile 1 & Ref & Ref & Ref & Ref & - & - & - & - \\
\hline Quartile 2 & 0.039 & 0.099 & 0.008 & 0.822 & - & - & - & - \\
\hline Quartile 3 & 0.005 & 0.832 & -0.038 & 0.256 & - & - & - & - \\
\hline Quartile 4 & -0.021 & 0.347 & -0.093 & 0.007 & - & - & - & - \\
\hline
\end{tabular}

${ }^{a}$ Adjusted for BMI, sex and smoking status

${ }^{\mathrm{b}}$ Adjusted for BMI, age and smoking status unit increase $=-0.072, p=0.002$ ), CRP (estimate per unit increase $=-0.032, p=0.025)$ and $\mathrm{HbA}_{1 \mathrm{c}}$ levels (estimate per unit increase $=-0.113, p<0.001)$ in continuous models. Shannon's diversity was also inversely associated with HOMA-IR (estimate per unit increase $=-0.047, p=0.001$ ) and $\mathrm{HbA}_{1 \mathrm{c}}$ levels (estimate per unit increase $=-0.042, p=0.003$ ) in NFBC1966. In TwinsUK, Shannon's diversity was inversely associated with HOMA-IR (estimate per unit increase = $-0.062, p<0.001$ ) and CRP (estimate per unit increase $=$ $-0.018, p=0.031$ ). The number of observed ASVs was also inversely associated with HOMA-IR (estimate per unit increase $=-0.106, p=0.001$ ) and CRP (estimate per unit increase $=-0.041, p=0.019$ ). In the NFBC1966, the association between BMI and the number of observed ASVs was no longer significant after adjustment for HOMA-IR ( $p$ value without HOMA-IR: $0.001, p$ value in model including HOMA-IR: 0.489 ) and CRP ( $p$ value in model including CRP: 0.082) (ESM Table 3). However, the association between BMI and the number of observed ASVs remained significant in multivariable models including $\mathrm{HbA}_{1 \mathrm{c}}$. In the TwinsUK cohort, BMI remained strongly inversely associated with alpha diversity following adjustments for HOMA-IR or CRP (ESM Table 3).

\section{Beta diversity}

HOMA-IR, CRP and $\mathrm{HbA}_{1 \mathrm{c}}$ were associated with all community composition measures except for weighted UniFrac and binomial deviance dissimilarity for CRP in the NFBC1966 (Table 3). Similar results were found for HOMA-IR in the TwinsUK cohort. However, CRP was only associated with Jaccard $(p=0.043)$ in TwinsUK. BMI was associated with all community composition measures except for weighted UniFrac in the NFBC1966 cohort and with all beta diversity measures in TwinsUK (ESM Table 4). However, the associations between BMI and microbial composition for binomial and unweighted UniFrac metrics were no longer significant after adjustment for HOMA-IR in NFBC1966 (ESM Table 4). BMI remained strongly associated with four beta diversity matrices, regardless of the inclusion of HOMA-IR or CRP in the model in the TwinsUK cohort.

\section{Relative abundance at the genus level}

In the NFBC1966 cohort, 16 individual genera met FDRadjusted significance level $(<0.05)$ for their associations with 
Table 3 Association of metabolic variables with community composition using measures of beta diversity in NFBC1966 and TwinsUK

\begin{tabular}{|c|c|c|c|c|c|c|c|c|}
\hline \multirow[b]{2}{*}{ Variable } & \multicolumn{4}{|c|}{$\operatorname{NFBC} 1966(\mathrm{n}=506)^{\mathrm{a}}$} & \multicolumn{4}{|c|}{ TwinsUK $(n=1140)^{b}$} \\
\hline & $\begin{array}{l}\text { Binomial } p \\
\text { value }\end{array}$ & $\begin{array}{l}\text { Jaccard } p \\
\text { value }\end{array}$ & $\begin{array}{l}\text { Weighted UniFrac } \\
p \text { value }\end{array}$ & $\begin{array}{l}\text { Unweighted } \\
\text { UniFrac } p \text { value }\end{array}$ & $\begin{array}{l}\text { Binomial } p \\
\text { value }\end{array}$ & $\begin{array}{l}\text { Jaccard } p \\
\text { value }\end{array}$ & $\begin{array}{l}\text { Weighted UniFrac } \\
p \text { value }\end{array}$ & $\begin{array}{l}\text { Unweighted } \\
\text { UniFrac } p \text { value }\end{array}$ \\
\hline HOMA-IR & $<0.001$ & $<0.001$ & 0.563 & 0.004 & $<0.001$ & $<0.001$ & 0.072 & $<0.001$ \\
\hline CRP & 0.112 & 0.007 & 0.384 & 0.047 & 0.345 & 0.043 & 0.241 & 0.099 \\
\hline $\mathrm{HbA}_{1 \mathrm{c}}$ & $<0.001$ & $<0.001$ & 0.112 & $<0.001$ & - & - & - & - \\
\hline
\end{tabular}

${ }^{\text {a }}$ Adjusted for BMI, sex and smoking status

${ }^{\mathrm{b}}$ Adjusted for BMI, age and smoking status

HOMA-IR, ten with CRP and nine with $\mathrm{HbA}_{1 \mathrm{c}}$. Nineteen individual genera met FDR-adjusted significance $(<0.05)$ with HOMA-IR and eight with CRP in the TwinsUK cohort. Higher values of HOMA-IR were strongly associated with higher mean relative abundances of members of the Peptococcaceae (estimate per unit increase $=11.62$, $p<0.001$ ), Bifidobacteriaceae (genus Gardnerella) (estimate per unit increase $=2.64, p<0.001)$, Veillonellaceae, Lachnospiraceae (genus Blautia) and Prevotellaceae (genus Paraprevotella) families and lower mean relative abundances of members from the Peptostreptococcaceae (estimate per unit increase $=-0.52, p<0.001$ ), Peptococcaceae (estimate per unit increase $=-0.23, p<0.001$ ), and Prevotellaceae (genus Prevotella) families (ESM Table 5) in the NFBC1966 cohort. Higher values of HOMA-IR were also associated with higher mean relative abundances of members from Lachnospiraceae (genus Blautia) and Prevotellaceae (genus UCG-003) and lower mean relative abundances of Prevotella in TwinsUK (ESM Table 6). Higher CRP was associated with lower mean relative abundances of members from the Paraprevotellaceae, Peptococcaceae, Veillonellaceae and Peptostreptococcaceae families and higher mean relative abundance of one genus, Peptococcus in NFBC1966 (ESM Table 5). Higher levels of $\mathrm{CRP}$ were associated with higher mean relative abundances of members from the Enterobacteriaceae, Elusimicrobiaceae, Erysipelotrichaceae and Prevotellaceae families and lower mean relative abundance of Dysgonomonas and NK4A214 group (family Ruminococcaceae) in TwinsUK (ESM Table 6). Higher levels of $\mathrm{HbA}_{1 \mathrm{c}}$ were associated with lower mean relative abundances of members from the Bifidobacteriaceae, Peptococcaceae, Veillonellaceae and Peptostreptococcaceae families and higher mean relative abundance of one genus, Oxalobacter, in the NFBC1966 cohort (ESM Table 5). The metabolic biomarkers were not associated with the presence/absence of individual genera except for the genus Gardneralla and $\mathrm{HbA}_{1 \mathrm{c}}$ (estimate per unit increase $=3.87, p=0.013$ ) in NFBC1966. Higher values of HOMA-IR were associated with higher presence of members of the Lachnospiraceae family and Veillonella in TwinsUK.

\section{Discussion}

In this analysis of gut microbiome profiles and metabolic variables in two population-based cohorts, higher levels of HOMA-IR, CRP and $\mathrm{HbA}_{1 \mathrm{c}}$ were associated with lower diversity of the gut microbiome, even after adjustment for BMI and other factors. In addition, HOMA-IR, CRP and $\mathrm{HbA}_{1 \mathrm{c}}$ were associated with differences in microbial community between individuals as indicated by associations with three beta diversity measures, but were not associated with weighted UniFrac, suggesting that the metabolic biomarkers were associated with differences in the presence or absence of bacteria in different communities but not with differences in abundance.

The unweighted UniFrac measure allows the detection of differences in the presence or absence of lineages of bacteria in different communities. On the other hand, the weighted UniFrac measure helps to detect differences in abundance even when the overall groups of organisms that are present in each sample remain the same [31]. In weighted UniFrac, low-abundance taxa have a much lower weight than in unweighted UniFrac and so will have a lower impact on the total distance reported by the metric. Further, HOMA-IR, $\mathrm{CRP}$ and $\mathrm{HbA}_{1 \mathrm{c}}$ were mainly associated with the relative abundance of specific genera, and less with their presence or absence, indicating that the associations between the metabolic biomarkers and microbiome diversity captured by beta diversity measures were potentially driven by a consortium of bacteria present at low relative abundance. In addition, although only observed in the NFBC1966 cohort, in an exploratory analysis we found that the association of BMI and microbiome diversity was attenuated when adjusting the model for HOMA-IR, supporting that some degree of the BMI-microbiome relation might be explained by the association of insulin resistance with microbial diversity.

Our data support the hypothesis that greater gut microbiome diversity is associated with better insulin sensitivity [6]. A clinical trial studying the effect of faecal transplant from lean donors to men diagnosed with the metabolic syndrome found that insulin sensitivity had improved and gut 
bacterial diversity had increased 6 weeks post transplantation [32]. These results corroborate other studies identifying gut microbial alterations and attenuation of the metabolic syndrome after various weight-loss interventions [33, 34]. As described previously, the gut microbiome can trigger inflammatory processes associated with obesity and insulin resistance by stimulating immune cells through lipopolysaccharides derived from bacterial membranes $[35,36]$. Furthermore, microbial-derived short-chain fatty acids, including butyrate [37], can enhance insulin sensitivity [38] and suppress insulin-mediated fat accumulation [39]. CRP is a marker of chronic low-grade systemic inflammation associated with obesity and insulin resistance [40]. Previous studies have found a similar relationship between systematic inflammation, through analysis of high-sensitivity CRP plasma levels and reduced bacterial diversity, supporting our findings [41]. Our results suggest that higher levels of $\mathrm{HbA}_{1 \mathrm{c}}$ were also associated with lower bacterial diversity. Existing literature on the association between $\mathrm{HbA}_{1 \mathrm{c}}$ level and gut microbiome composition are inconsistent. Some studies reported evidence of an association between $\mathrm{HbA}_{1 \mathrm{c}}$ level and bacterial group counts [42] while others did not find any relationship [43]. These findings could be explained by the fact that elevated insulin levels or HOMA-IR appeared to identify certain traits of the metabolic syndrome, especially abdominal obesity, earlier than that seen in changes to both $\mathrm{HbA}_{1 \mathrm{c}}$ and measures of glucose [44].

Consistent with other studies, BMI was inversely associated with gut microbiome diversity in this study [13]. However, following adjustment for HOMA-IR and CRP, the associations between BMI and some measures of alpha- and beta diversity were no longer detected, suggesting that the observed relationship between BMI and gut microbiome diversity might be indirect and influenced by measures of insulin resistance and inflammation. This hypothesis needs to be further explored in other cohorts as results from TwinsUK suggested that associations between BMI and gut microbial diversity were attenuated but remained significant.

The relative abundance of individual genera was associated with HOMA-IR (16 and 19 genera in NFBC1966 and TwinsUK, respectively), with CRP (ten and eight genera in NFBC1966 and TwinsUK, respectively) and with $\mathrm{HbA}_{1 \mathrm{c}}$ (nine genera in NFBC1966). Overall, the GAMLSS models showed that metabolic biomarkers were more associated with the relative abundance of the taxa rather than their presence or absence. At the genus level, Blautia was positively and Prevotella inversely associated with HOMA-IR in both cohorts. In previous studies, Blautia has been reported to be related to disturbances in glucose metabolism and type 2 diabetes [45-48]. At the mechanistic level, exposure of human tissue to Blautia species has been shown to induce an inflammatory response greater than that induced by lipopolysaccharide stimulation [49]. In addition, in a rat diabetes model, exposure to Blautia was positively correlated with inflammatory indicators including, IL-1 $\beta$, TNF- $\alpha$, IL- 6 and lipopolysaccharides [50]. These results suggest that Blautia might be related to metabolic health through its effects on specific inflammatory pathways. Lower abundance of Prevotella in diabetic patients was previously observed, consistent with our data on insulin resistance and metabolic health [51-53]. In addition, Prevotella was shown to be positively associated with dietary fibre-induced improvement in glucose metabolism by potentially playing a role in the fermentation of complex polysaccharides and in the storage of glycogen [54]. In contrast, increased abundance of Prevotella strains were associated with insulin resistance in a non-diabetic cohort [11] with obesity $[55,56]$ and with low-grade inflammation in the gut [57]. However, it was speculated that these effects may not be causally linked to the presence of Prevotella and that other members of the Prevotella-dominated microbiome may have the propensity to promote inflammation [58] Overall, these findings indicate that Blautia and Prevotella may induce biological changes particularly in inflammatory pathways that may explain their association with metabolic health in our study. Further experimental studies are needed to identify mechanisms by which these bacteria may be related to host metabolic health.

A limitation of our study was the cross-sectional nature of the analyses. Repeated, prospectively collected samples are now needed to identify potential causal relationships between gut microbiome and markers of metabolic health and to assess their respective association with risk of diseases related to metabolic dysfunction such as type 2 diabetes or certain cancers. Other limitations were that TwinsUK only included female participants, although sensitivity analyses restricted to women in the NFBC1966 showed similar results. Further, data on $\mathrm{HbA}_{1 \mathrm{c}}$ were not available in TwinsUK to validate the NFBC1966 results. We also note differences between the studied NFBC1966 and TwinsUK populations in terms of age, obesity prevalence and metabolic variables. However, being able to observe consistent associations between gut microbiome composition and measures of metabolic health in two different populations and independently of other potential factors such as age and obesity may indicate the results are generalisable across different populations. An additional limitation is that the observed associations of microbiome diversity measures with metabolic health biomarkers in both cohorts may have also been inflated or attenuated by unmeasured or poorly measured confounders, such as dietary and fibre intake and the use of medication. Finally, we recognise the limited taxonomic resolution and accuracy of the 16S rRNA gene sequencing methodology, preventing us from adequately performing species-level associative analysis and resulting in a limited overlap between the two cohorts when focusing on associations with individual genera. To unambiguously discriminate low-abundance taxa 
from noise and further replication and extension (e.g., metagenomics) of results in other population-based studies is warranted.

Our study suggests that general population cohorts are valuable in identifying potential associations between microbial features and measures of metabolic health. However, the use of cross-sectional data does not allow us to causally interpret these associations, since interactions between metabolic biomarkers and the gut microbiome are complex and dynamic and can be strongly affected by behavioural changes. Therefore, it is now critical for future studies to collect longitudinal data of both lifestyle exposures and the microbiome to help understand the dynamic relationship between the gut microbiome and host metabolism. Integration of microbiome data paired with faecal metabolomics data may also provide a more complete picture of the metabolic microbial mechanisms that contribute to metabolic balance between the host and the gut microbiome.

In conclusion, markers of insulin resistance, poor control of blood glucose levels and systemic inflammation were associated with lower gut microbiome diversity and distinct microbial community structures in both the NFBC1966 and TwinsUK cohorts, even after adjustment for BMI and other variables. These results from two distinct population-based cohorts provide evidence that individuals with worse metabolic control have lower gut microbial diversity. It is, however, impossible to conclude if these differences in microbial composition and taxa associations are a cause or a consequence of metabolic dysfunction. Thus, large-scale, prospective studies with collection of faecal samples and longitudinal data on lifestyle and metabolic biomarkers at several timepoints are now needed to validate and extend these observations.

Supplementary Information The online version contains peer-reviewed but unedited supplementary material available at https://doi.org/10.1007/ s00125-021-05464-w.

Acknowledgements We wish to thank all the NFBC1966 and TwinsUK participants, and research personnel who have participated in the data collection.

Data availability The datasets used and/or statistical analysis code for the current study are available from the authors (Marc Gunter, gunterm@iarc.fr) on reasonable request.

Funding This work was supported by the International Agency for Research on Cancer, WHO, France and the National Cancer Institute, USA.

Authors' relationships and activities Where authors are identified as personnel of the International Agency for Research on Cancer/WHO, the authors alone are responsible for the views expressed in this article and they do not necessarily represent the decisions, policy or views of the International Agency for Research on Cancer/WHO.
Contribution statement K-HH, M-RJ contributed to the conception and design, and acquisition of data for the NFBC1966. SZ, IH, MJG conducted the analysis of the NFBC1966 data, and the interpretation of the NFBC1966 and TwinsUK data. VV, EL, EV, RS provided support with the NFBC1966 data analysis. PW and CJS prepared the ASVs data in the TwinsUK cohort. PL conducted the analysis of the TwinsUK data. CM provided support with the TwinsUK data analysis. SZ drafted the manuscript. All authors revised the manuscript critically for important intellectual content. All authors read and approved the final version to be published. SZ is responsible for the integrity of the work as a whole.

Open Access This article is licensed under a Creative Commons Attribution 4.0 International License, which permits use, sharing, adaptation, distribution and reproduction in any medium or format, as long as you give appropriate credit to the original author(s) and the source, provide a link to the Creative Commons licence, and indicate if changes were made. The images or other third party material in this article are included in the article's Creative Commons licence, unless indicated otherwise in a credit line to the material. If material is not included in the article's Creative Commons licence and your intended use is not permitted by statutory regulation or exceeds the permitted use, you will need to obtain permission directly from the copyright holder. To view a copy of this licence, visit http://creativecommons.org/licenses/by/4.0/.

\section{References}

1. Jaacks LM, Vandevijvere S, Pan A et al (2019) The obesity transition: stages of the global epidemic. Lancet Diabetes Endocrinol 7(3):231-240. https://doi.org/10.1016/S2213-8587(19)30026-9

2. Zheng Y, Ley SH, Hu FB (2018) Global aetiology and epidemiology of type 2 diabetes mellitus and its complications. Nat Rev Endocrinol 14(2):88-98. https://doi.org/10.1038/nrendo.2017.151

3. Taylor R (2012) Insulin Resistance and Type 2 Diabetes. Diabetes 61(4):778-779. https://doi.org/10.2337/db12-0073

4. Kahn SE, Hull RL, Utzschneider KM (2006) Mechanisms linking obesity to insulin resistance and type 2 diabetes. Nature 444(7121): 840-846. https://doi.org/10.1038/nature05482

5. Owei I, Umekwe N, Provo C, Wan J, Dagogo-Jack S (2017) Insulin-sensitive and insulin-resistant obese and non-obese phenotypes: role in prediction of incident pre-diabetes in a longitudinal biracial cohort. BMJ Open Diabetes Res Care 5(1):e000415. https://doi.org/10.1136/bmjdrc-2017-000415

6. Le Chatelier E, Nielsen T, Qin J et al (2013) Richness of human gut microbiome correlates with metabolic markers. Nature 500(7464): 541-546. https://doi.org/10.1038/nature12506

7. Larsen N, Vogensen FK, van den Berg FWJ et al (2010) Gut microbiota in human adults with type 2 diabetes differs from nondiabetic adults. PLoS One 5(2):e9085. https://doi.org/10.1371/ journal.pone.0009085

8. Qin J, Li Y, Cai Z et al (2012) A metagenome-wide association study of gut microbiota in type 2 diabetes. Nature 490(7418):5560. https://doi.org/10.1038/nature1 1450

9. Lee CJ, Sears CL, Maruthur N (2020) Gut microbiome and its role in obesity and insulin resistance. Ann N Y Acad Sci 1461(1):37-52. https://doi.org/10.1111/nyas.14107

10. Lee CC, Watkins SM, Lorenzo C et al (2016) Branched-Chain Amino Acids and Insulin Metabolism: The Insulin Resistance Atherosclerosis Study (IRAS). Diabetes Care 39(4):582-588. https://doi.org/10.2337/dc15-2284 
11. Pedersen HK, Gudmundsdottir V, Nielsen HB et al (2016) Human gut microbes impact host serum metabolome and insulin sensitivity. Nature 535(7612):376-381. https://doi.org/10.1038/nature18646

12. Rantakallio P (1988) The longitudinal study of the northern Finland birth cohort of 1966. Paediatr Perinat Epidemiol 2(1):59-88. https:// doi.org/10.1111/j.1365-3016.1988.tb00180.x

13. Loftfield E, Herzig K-H, Caporaso JG et al (2020) Association of body mass index with fecal microbial diversity and metabolites in the northern Finland birth cohort. Cancer Epidemiol Biomarkers Prev 29(11):2289-2299

14. Perkiömäki N, Auvinen J, Tulppo MP et al (2016) Association between Birth Characteristics and Cardiovascular Autonomic Function at Mid-Life. PLoS One 11(8):e0161604. https://doi.org/ 10.1371/journal.pone.0161604

15. Matthews DR, Hosker JP, Rudenski AS, Naylor BA, Treacher DF, Turner RC (1985) Homeostasis model assessment: insulin resistance and beta-cell function from fasting plasma glucose and insulin concentrations in man. Diabetologia 28(7):412-419. https://doi. org $/ 10.1007 / \mathrm{bf00280883}$

16. Vogtmann E, Chen J, Amir A et al (2017) Comparison of Collection Methods for Fecal Samples in Microbiome Studies. Am J Epidemiol 185(2):115-123. https://doi.org/10.1093/aje/ kww177

17. Caporaso JG, Lauber CL, Walters WA et al (2012) Ultra-highthroughput microbial community analysis on the Illumina HiSeq and MiSeq platforms. ISME J 6(8):1621-1624. https://doi.org/10. 1038/ismej.2012.8

18. Sinha R, Abu-Ali G, Vogtmann E et al (2017) Assessment of variation in microbial community amplicon sequencing by the Microbiome Quality Control (MBQC) project consortium. Nat Biotechnol 35(11):1077-1086. https://doi.org/10.1038/nbt.3981

19. Bolyen E, Rideout JR, Dillon MR et al (2019) Reproducible, interactive, scalable and extensible microbiome data science using QIIME 2. Nat Biotechnol 37(8):852-857. https://doi.org/10.1038/ s41587-019-0209-9

20. Callahan BJ, McMurdie PJ, Rosen MJ, Han AW, Johnson AJA, Holmes SP (2016) DADA2: High-resolution sample inference from Illumina amplicon data. Nat Methods 13(7):581-583. https:/doi. org/10.1038/nmeth.3869

21. Bokulich NA, Kaehler BD, Rideout JR et al (2018) Optimizing taxonomic classification of marker-gene amplicon sequences with QIIME 2's q2-feature-classifier plugin. Microbiome 6(1):90. https://doi.org/10.1186/s40168-018-0470-z

22. DeSantis TZ, Hugenholtz P, Larsen N et al (2006) Greengenes, a chimera-checked 16S rRNA gene database and workbench compatible with ARB. Appl Environ Microbiol 72(7):5069-5072. https:// doi.org/10.1128/AEM.03006-05

23. Katoh K, Standley DM (2013) MAFFT multiple sequence alignment software version 7: improvements in performance and usability. Mol Biol Evol 30(4):772-780. https://doi.org/10.1093/molbev/ mst010

24. Price MN, Dehal PS, Arkin AP (2010) FastTree 2 - approximately maximum-likelihood trees for large alignments. PLoS One 5(3): e9490. https://doi.org/10.1371/journal.pone.0009490

25. Oksanen J, Blanchet FG, Friendly M et al (2019) vegan: Community Ecology Package

26. Verdi S, Abbasian G, Bowyer RCE et al (2019) TwinsUK: The UK Adult Twin Registry Update. Twin Res Hum Genet 22(6):523-529. https://doi.org/10.1017/thg.2019.65

27. Jamshidi Y, Snieder H, Wang X, Spector TD, Carter ND, O’Dell SD (2006) Common polymorphisms in SOCS3 are not associated with body weight, insulin sensitivity or lipid profile in normal female twins. Diabetologia 49(2):306-310. https://doi.org/10. 1007/s00125-005-0093-3

28. Goodrich JK, Davenport ER, Beaumont M et al (2016) Genetic Determinants of the Gut Microbiome in UK Twins. Cell Host
Microbe 19(5):731-743. https://doi.org/10.1016/j.chom.2016.04. 017

29. Zhao N, Chen J, Carroll IM et al (2015) Testing in MicrobiomeProfiling Studies with MiRKAT, the Microbiome RegressionBased Kernel Association Test. Am J Hum Genet 96(5):797-807. https://doi.org/10.1016/j.ajhg.2015.04.003

30. Rigby RA, Stasinopoulos DM (2005) Generalized additive models for location, scale and shape. J R Stat Soc: Ser C: Appl Stat 54(3): 507-554. https://doi.org/10.1111/j.1467-9876.2005.00510.x

31. Lozupone CA, Hamady M, Kelley ST, Knight R (2007) Quantitative and Qualitative $\beta$ Diversity Measures Lead to Different Insights into Factors That Structure Microbial Communities. Appl Environ Microbiol 73(5):1576-1585. https:// doi.org/10.1128/AEM.01996-06

32. Vrieze A, Van Nood E, Holleman F et al (2012) Transfer of intestinal microbiota from lean donors increases insulin sensitivity in individuals with metabolic syndrome. Gastroenterology 143(4): 913-916.e7. https://doi.org/10.1053/j.gastro.2012.06.031

33. Li G, Xie C, Lu S et al (2017) Intermittent Fasting Promotes White Adipose Browning and Decreases Obesity by Shaping the Gut Microbiota. Cell Metab 26(4):672-685.e4. https://doi.org/10. 1016/j.cmet.2017.08.019

34. Wang L, Li P, Tang Z, Yan X, Feng B (2016) Structural modulation of the gut microbiota and the relationship with body weight: compared evaluation of liraglutide and saxagliptin treatment. Sci Rep 6(1):1-10. https://doi.org/10.1038/srep33251

35. Tremaroli V, Bäckhed F (2012) Functional interactions between the gut microbiota and host metabolism. Nature 489(7415):242-249. https://doi.org/10.1038/nature11552

36. Saad MJA, Santos A, Prada PO (2016) Linking Gut Microbiota and Inflammation to Obesity and Insulin Resistance. Physiology 31(4): 283-293. https://doi.org/10.1152/physiol.00041.2015

37. Rivière A, Selak M, Lantin D, Leroy F, De Vuyst L (2016) Bifidobacteria and Butyrate-Producing Colon Bacteria: Importance and Strategies for Their Stimulation in the Human Gut. Front Microbiol 7:979. https://doi.org/10.3389/fmicb.2016. 00979

38. Tolhurst G, Heffron H, Lam YS et al (2012) Short-Chain Fatty Acids Stimulate Glucagon-Like Peptide-1 Secretion via the GProtein-Coupled Receptor FFAR2. Diabetes 61(2):364-371. https://doi.org/10.2337/db11-1019

39. Kimura I, Ozawa K, Inoue D et al (2013) The gut microbiota suppresses insulin-mediated fat accumulation via the short-chain fatty acid receptor GPR43. Nat Commun 4:1829. https://doi.org/ $10.1038 /$ ncomms 2852

40. Uemura H, Katsuura-Kamano S, Yamaguchi M et al (2017) Relationships of serum high-sensitivity C-reactive protein and body size with insulin resistance in a Japanese cohort. PLoS One 12(6): e0178672. https://doi.org/10.1371/journal.pone.0178672

41. Verdam FJ, Fuentes S, de Jonge C et al (2013) Human intestinal microbiota composition is associated with local and systemic inflammation in obesity. Obesity 21(12):E607-E615. https://doi. org/10.1002/oby.20466

42. Leiva-Gea I, Sánchez-Alcoholado L, Martín-Tejedor B et al (2018) Gut Microbiota Differs in Composition and Functionality Between Children With Type 1 Diabetes and MODY2 and Healthy Control Subjects: A Case-Control Study. Diabetes Care 41(11):2385-2395. https://doi.org/10.2337/dc18-0253

43. Lambeth SM, Carson T, Lowe J et al (2015) Composition, Diversity and Abundance of Gut Microbiome in Prediabetes and Type 2 Diabetes. J Diabetes Obes 2(3):1-7. https://doi.org/10. 15436/2376-0949.15.031

44. Saravia G, Civeira F, Hurtado-Roca Y et al (2015) Glycated Hemoglobin, Fasting Insulin and the Metabolic Syndrome in Males. Cross-Sectional Analyses of the Aragon Workers' Health 
Study Baseline. PLoS One 10(8):e0132244. https://doi.org/10. 1371/journal.pone.0132244

45. Zhang X, Shen D, Fang Z et al (2013) Human gut microbiota changes reveal the progression of glucose intolerance. PLoS One 8(8):e71108. https://doi.org/10.1371/journal.pone.0071108

46. Lippert K, Kedenko L, Antonielli L et al (2017) Gut microbiota dysbiosis associated with glucose metabolism disorders and the metabolic syndrome in older adults. Benefic Microbes 8(4):545556. https://doi.org/10.3920/BM2016.0184

47. Egshatyan L, Kashtanova D, Popenko A et al (2016) Gut microbiota and diet in patients with different glucose tolerance. Endocr Connect 5(1):1-9. https://doi.org/10.1530/EC-15-0094

48. Kashtanova DA, Tkacheva ON, Doudinskaya EN et al (2018) Gut Microbiota in Patients with Different Metabolic Statuses: Moscow Study. Microorganisms 6(4):98. https://doi.org/10.3390/ microorganisms6040098

49. Tuovinen E, Keto J, Nikkilä J, Mättö J, Lähteenmäki K (2013) Cytokine response of human mononuclear cells induced by intestinal Clostridium species. Anaerobe 19:70-76. https://doi.org/10. 1016/j.anaerobe.2012.11.002

50. Zhu L, Sha L, Li K et al (2020) Dietary flaxseed oil rich in omega-3 suppresses severity of type 2 diabetes mellitus via antiinflammation and modulating gut microbiota in rats. Lipids Health Dis 19(1):20. https://doi.org/10.1186/s12944-019-1167-4

51. Krych $€$, Nielsen DS, Hansen AK, Hansen CHF (2015) Gut microbial markers are associated with diabetes onset, regulatory imbalance, and IFN- $\gamma$ level in NOD Mice. Gut Microbes 6(2):101-109. https://doi.org/10.1080/19490976.2015.1011876

52. Brown CT, Davis-Richardson AG, Giongo A et al (2011) Gut microbiome metagenomics analysis suggests a functional model for the development of autoimmunity for type 1 diabetes. PLoS One 6(10):e25792. https://doi.org/10.1371/journal.pone.0025792

53. Mejía-León ME, Petrosino JF, Ajami NJ, Domínguez-Bello MG, de la Barca AMC (2014) Fecal microbiota imbalance in Mexican children with type 1 diabetes. Sci Rep 4(1):3814. https://doi.org/10. 1038/srep03814

54. Kovatcheva-Datchary P, Nilsson A, Akrami R et al (2015) Dietary Fiber-Induced Improvement in Glucose Metabolism Is Associated with Increased Abundance of Prevotella. Cell Metab 22(6):971982. https://doi.org/10.1016/j.cmet.2015.10.001

55. Zhu L, Baker SS, Gill C et al (2013) Characterization of gut microbiomes in nonalcoholic steatohepatitis (NASH) patients: a connection between endogenous alcohol and NASH. Hepatology 57(2):601-609. https://doi.org/10.1002/hep.26093

56. Hu H-J, Park S-G, Jang HB et al (2015) Obesity Alters the Microbial Community Profile in Korean Adolescents. PLoS One 10(7):e0134333. https://doi.org/10.1371/journal.pone.0134333

57. Leite AZ, de Campos Rodrigues N, Gonzaga MI et al (2017) Detection of Increased Plasma Interleukin-6 Levels and Prevalence of Prevotella copri and Bacteroides vulgatus in the Feces of Type 2 Diabetes Patients. Front Immunol 8:1107. https:// doi.org/10.3389/fimmu.2017.01107

58. Iljazovic A, Roy U, Gálvez EJC et al (2021) Perturbation of the gut microbiome by Prevotella spp. enhances host susceptibility to mucosal inflammation. Mucosal Immunol 14(1):113-124. https:// doi.org/10.1038/s41385-020-0296-4

Publisher's note Springer Nature remains neutral with regard to jurisdictional claims in published maps and institutional affiliations. 